\title{
Das deutschlandweite, webbasierte ParaReg-Register zur lebenslangen Dokumentation von Querschnittgelähmten - Datenmodell, rechtlich-ethische Voraussetzungen und technische Implementierung
}

\author{
Germany-wide, Web-based ParaReg Registry for Lifelong \\ Monitoring of People with Spinal Cord Injury: Data Model, \\ Ethico-legal Prerequisites and Technical Implementation
}

Autoren

Rüdiger Rupp ${ }^{10}$, Patrick Jersch¹, Christian Schuld ${ }^{1}$ (D) Joachim Schweidler ${ }^{1}$, Nils-Hendrik Benning ${ }^{2}$, Petra Knaup-Gregori' ${ }^{2}$, Mirko Aach ${ }^{3}$, Andreas Badke ${ }^{4}$, Andreas Hildesheim ${ }^{5}$, Doris Maier ${ }^{6}$, Norbert Weidner ${ }^{1}$, Marion Saur ${ }^{7}$

Institute

1 Klinik für Paraplegiologie, Universitätsklinikum Heidelberg, Heidelberg, Deutschland

2 Institut für Medizinische Informatik, Universitätsklinikum Heidelberg, Heidelberg, Deutschland

3 Abteilung für Rückenmarksverletzte, Berufsgenossenschaftliches Universitatsklinikum Bergmannsheil, Bochum, Deutschland

4 Abteilung für Querschnittgelähmte, Berufsgenossenschaftliche Klinik Tübingen, Tübingen, Deutschland

5 Querschnittzentrum, Neurologisches Rehabilitationszentrum Godeshöhe e. V., Bonn, Deutschland

6 Zentrum für Rückenmarkverletzte, Berufsgenossenschaftliche Unfallklinik Murnau, Murnau, Deutschland

7 Zentrum für Tetra-/Paraplegie, Orthopädische Klinik Hessisch Lichtenau, Hessisch Lichtenau, Deutschland

Schlüsselwörter

Querschnittlähmung, Register, patientenzentriert, DSGVO, Flask-Framework

Key words

Spinal cord injury, registry, GDPR, patient-centered,

Flask framework

\section{Bibliografie}

Gesundheitswesen 2021; 83 (Suppl. 1): S18-S26

DOI 10.1055/a-1538-6537

ISSN 0949-7013

(c) 2021. Thieme. All rights reserved.

Georg Thieme Verlag, Rüdigerstraße 14,

70469 Stuttgart, Germany
Korrespondenzadresse

Rüdiger Rupp

Klinik für Paraplegiologie

Universitätsklinikum Heidelberg

Schlierbacher Landstrasse 200a

69118 Heidelberg

Deutschland

ruediger.rupp@med.uni-heidelberg.de

\section{ABSTRACT}

Ziel der Studie In Deutschland unterscheiden sich die Behandlungspfade von frisch Querschnittgelähmten in Abhängigkeit von intrinsisch-krankheitsspezifischen und extrinsischen Faktoren erheblich. Welche dieser Faktoren mit einem verbesserten Outcome, weniger Folgekomplikationen und stationären Wiederaufnahmen assoziiert sind, ist bis heute nicht bekannt. Daher soll das deutschlandweite, patientenzentrierte, webbasierte ParaReg-Register implementiert werden, um langfristig eine bessere Qualität der Patientenversorgung, Planung der Behandlungspfade und Kosteneffizienz zu erreichen.

Methodik In der Konzeptionierungsphase 2017/18 wurde das Datenmodell des Registers vom ParaReg-Leitungskomitee in einem iterativen Prozess zusammen mit dem erweiterten Vorstand der Deutschsprachigen Medizinischen Gesellschaft für Paraplegiologie e.V. (DMGP) und Patientenvertretern entwickelt. In ParaReg werden soziale und medizinische Routinedaten zusammen mit international etablierten neurologischen, funktionellen und partizipationsbezogenen Scores dokumentiert. Die Vergabe einer eindeutigen Patienten-ID erlaubt lebenslang eine zentrumsübergreifende Dokumentation von stationären Aufenthalten in einem der 27 in Deutschland in der DMGP organisierten Querschnittzentren. Das ParaReg-Datenschutzkonzept und die Patienteninformation/-einwilligung orientieren sich an den um DSGVO-relevante Aspekte erweiterten Vorlagen des Open Source Registers für seltene Erkrankungen (OSSE). 
Ergebnisse In der 2019 begonnenen Realisierungsphase wurde die informationstechnische Infrastruktur gemäß des klinischen ID-Management-Moduls der Technologie- und Methodenplattform für die vernetzte medizinische Forschung e.V. (TMF) umgesetzt. Parallel wurden die rechtlich-ethischen Voraussetzungen für den Registerbetrieb unter der Schirmherrschaft der DMGP geschaffen. In das Datenschutzkonzept sind die Empfehlungen der Arbeitsgruppe Datenschutz der TMF eingeflossen. Basierend auf den Rückmeldungen aus der AlphaTestphase mit Eingabe der Aufenthaltsdaten von 40 Patienten wurde die Ergonomie der elektronischen Eingabeformulare speziell für mobile Eingabegeräte verbessert.

Schlussfolgerung Mit Abschluss der monozentrischen AlphaTestphase hat die multizentrische Datenerhebung an 5 DMGPQuerschnittzentren begonnen. Die Nachhaltigkeit von ParaReg ist durch die strukturelle und finanzielle Unterstützung durch die DMGP auch nach Auslaufen der Förderung durch das Bundesministerium für Bildung und Forschung (BMBF) gesichert.

\section{ABSTRACT}

Objective In Germany, treatment paths for patients with acute spinal cord injury ( $\mathrm{SCl}$ ) differ considerably depending on intrinsic, disease-specific and extrinsic factors. Which of these factors are associated with improved outcome with fewer subsequent complications and inpatient re-admissions is not clear. The German-wide, patient-centered, web-based ParaReg registry will be implemented to improve the long-term quality of patient care and the planning of treatment paths with increased cost-effectiveness.
Methods In the 2017-18 conceptualization phase, the data model of the registry was developed in an iterative process of the ParaReg steering committee together with the extended DMGP board and patient representatives. In ParaReg, routine social and medical data as well as internationally established neurological, functional and participation scores will be documented. The assignment of a unique patient ID allows a lifelong, cross-center documentation of inpatient stays in one of the $27 \mathrm{SCl}$ centers organized in the German-speaking Medical Society for SCI (DMGP). The ParaReg data protection concept and patient information/consent are based on the Open Source Registry for Rare Diseases (OSSE) which were extended by GDPR-relevant aspects.

Results In the realization phase, which started in 2019, the information technology infrastructure was implemented according to the clinical ID management module of the Technology and Methods Platform for Networked Medical Research (TMF). In parallel, the legal and ethical prerequisites for registry operation under the patronage of the DMGP were created. Recommendations of the working group data protection of the TMF were integrated into ParaReg's data protection concept. Based on the feedback from the alpha test phase with documentation of the hospitalization data of 40 patients, the ergonomics of the electronic case report forms were improved in particular for data entry on mobile devices.

Conclusion After completion of the monocentric alpha test phase, the multicenter data acquisition was started in 5 DMGP$\mathrm{SCl}$ centers. The sustainability of ParaReg is ensured by the structural and financial support of the DMGP after expiry of the funding by the German Federal Ministry of Education and Research (BMBF).

\section{Einleitung}

In Deutschland treten nach aktuellen Schätzungen jährlich 2300 frische Rückenmarkverletzungen auf, wobei von einer Gesamtpopulation von 140000 Querschnittgelähmten ausgegangen wird [1]. Eine Rückenmarksschädigung kann sowohl durch traumatische (Wirbelsäulenverletzungen) als auch durch nicht-traumatische Ursachen (Raumforderungen, Ischämien, Infektionen) entstehen. Der initiale Behandlungspfad von Patienten mit einer neu erworbenen Querschnittlähmung wird im Wesentlichen von intrinsisch-krankheitsbedingten Faktoren bestimmt: Während Patienten nach einem Unfall oder einem Sturz zuerst in einem chirurgischen (Trauma-/Wirbelsäulenchirurgie) Zentrum versorgt werden, findet die Erstbehandlung bei einer krankheitsbedingten (z. B. Entzündungen, Tumor, Durchblutungsproblem) Querschnittlähmung in einer nicht operativ ausgerichteten (z. B. neurologische oder Neurorehabilitationsklinik) Einrichtung statt. Der weitere stationäre Behandlungsverlauf wird dann aber nicht nur durch intrinsische, sondern auch durch extrinsische Faktoren wie der Kostenträgerzuständigkeit (gesetzliche/private Krankenversicherung, gesetzliche Unfallversicherung) oder Verfügbarkeit von Therapieplätzen bestimmt. Nach der stationären Entlassung werden Unterschiede bei der Versorgung besonders deutlich, da eine lebenslange ambulan- te Nachsorge zur Behandlung von typischen mit einer Querschnittlähmung einhergehenden Komplikationen wie Hautschädigungen (Dekubitus), Lungenentzündungen, Spastik, urologische Komplikationen, oder Darmprobleme ausschließlich von spezialisierten Querschnittzentren angeboten wird ( $\triangleright$ Abb. 1). Zusammenfassend gibt es also eine Reihe von Unterschieden in der stationären und ambulanten Versorgung von Menschen mit einer Querschnittlähmung, die die neurologische und funktionelle Erholung, die Häufigkeit von Komplikationen, und letztlich die Selbständigkeit sowie Teilhabe und Lebensqualität beeinflussen. Bisher gibt es allerdings keine strukturierte Datensammlung zur Untersuchung dieser Zusammenhänge.

Daher soll mit Förderung durch das Bundesministerium für Bildung und Forschung (BMBF) und der Deutschsprachigen Medizinischen Gesellschaft für Paraplegiologie e.V. (DMGP) das deutschlandweite, patientenzentrierte, webbasierte ParaReg-Register zur Dokumentation von medizinischen, sozialen und partizipationsbezogen Daten von Querschnittgelähmten aufgebaut werden. Das vorrangige Ziel des ParaReg-Registers besteht in der Identifikation von Faktoren, die mit einem schlechteren Outcome, häufigen Komplikationen und ggfs. stationären Aufenthalten assoziiert sind. Langfristig soll mit den Daten aus ParaReg die Qualität der Patientenversorgung, 


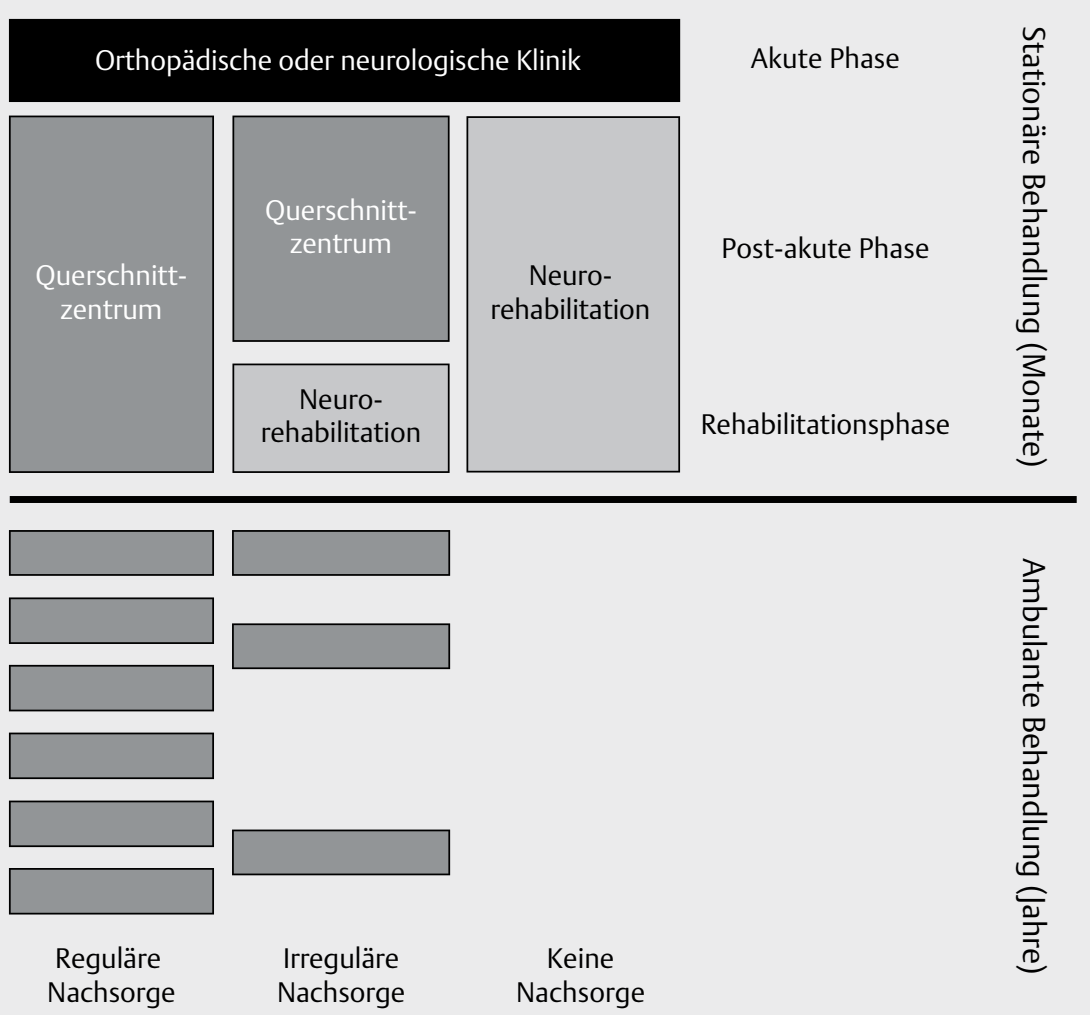

-Abb. 1 Unterschiedliche Behandlungspfade nach frisch eingetretener Querschnittlähmung in Deutschland.

die Therapieplanung und Steuerung des Behandlungspfades unter Berücksichtigung der Kosteneffizienz verbessert werden.

\section{Methodik}

\section{Datenmodell}

Im ParaReg-Register wird jeder stationäre und später auch ambulante Aufenthalt eines Patienten mit einer akuten oder chronischen Querschnittlähmung in einem DMGP-assoziierten Querschnittzentrum (insgesamt 27 in Deutschland) dokumentiert. Das ParaRegDatenmodell wurde in einem iterativen Prozess vom ParaReg-Leitungskomitee (Autoren dieser Arbeit) während der vom BMBF geförderten Konzeptionierungsphase in den Jahren 2017/18 entwickelt und besteht aus einem Minimalsatz aus Variablen und Assessments, die neben der primären Ursache und Ätiologie der Querschnittlähmung auch sozio-medizinische und komplikationsassoziierte Variablen zusammen mit etablierten funktionellen und neurologischen Assessments beschreiben ( Tab. 1).

Bei jedem Aufenthalt wird die Hauptdiagnose zusammen mit den drei wichtigsten Nebendiagnosen und vorbestehenden Komorbiditäten dokumentiert. Die Dokumentation des neurologischen und funktionellen Patientenstatus erfolgt über die International Standards for Neurological Classification of Spinal Cord Injury (ISNCSCI) [2] und den Spinal Cord Independence Measure (SCIM) III [3] (bei chronischen Patienten als Self-Report-Variante [4]). Generell wurden die Variablen des ParaReg-Datenmodells entspre- chend ihrer routinemäßigen Verfügbarkeit in den Krankenakten ausgewählt. Neben dem ISNCSCI und dem SCIM III gewährleistet die Implementierung der International Data Sets on SCI der International Spinal Cord Society (ISCOS) die Kompatibilität des ParaReg-Datenmodells mit internationalen Querschnitt-Registern [5]. Zusätzlich wurden die anwendbaren Elemente des Common Data Set des Open Source Registers für seltene Erkrankungen (OSSE; http://osse-register.de) integriert. Daten zu Partizipation und Lebensqualität werden über den World Health Organization Quality of Life Group - Abbreviated Version (WHOQOL-BREF) abgefragt [6]. Ein wesentlicher Fokus der Datenmodellentwicklung lag auf der Definition von Auswahllisten für alle Datenelemente zur Minimierung von schlecht zu analysierenden Freitexteingaben. Der Datensatz eines Patienten wurde in einen zwingend einzugebenden Kerndatensatz (Datum und Ursache der Querschnittlähmung, Tetra-/Paraplegie, Hauptdiagnose des Aufenthaltes) und einen gewünschten erweiterten Kerndatensatz mit insgesamt 234 Einzelfeldern pro Aufenthalt eingeteilt.

Das ParaReg-Datenmodell wurde abschließend durch 16 Mitglieder des erweiterten Vorstands der DMGP als auch durch 13 Peers der Fördergemeinschaft der Querschnittgelähmten e.V. (FGQ) geprüft und freigegeben.

\section{Organisations- und Rechtstruktur}

Das ParaReg-Register wird unter der Schirmherrschaft der DMGPFachgesellschaft entwickelt und betrieben. Im Jahr 2016 wurde innerhalb der DMGP eine Registergruppe durch den Vorstand der 
> Tab. 1 Übersicht der Variablenkategorien des ParaReg-Registers.

\begin{tabular}{|c|c|}
\hline Kategorie & Variablen \\
\hline $\begin{array}{l}\text { Persönliche Patientendaten } \\
\text { (Eingabe bei jedem } \\
\text { Aufenthalt zur } \\
\text { Identifikation) }\end{array}$ & $\begin{array}{l}\text { - Geschlecht } \\
\text { - Vorname } \\
\text { - Nachname } \\
\text { - Geburtsdatum } \\
\text { - Geburtsort } \\
\text { - Aktuelle Anschrift }\end{array}$ \\
\hline $\begin{array}{l}\text { Pseudonymisierte } \\
\text { Patientendaten (einmalige } \\
\text { Eingabe) }\end{array}$ & $\begin{array}{l}\text { - Geburtsjahr (berechnet aus dem Geburtstag) } \\
\text { - Datum der Studienzustimmung } \\
\text { - Datum und Art des Studienaustritts (wo anwendbar) } \\
\text { - Todesdatum (wo anwendbar) } \\
\text { - Todesursache } \\
\text { - Ort (Institution, zu Hause) des Todes }\end{array}$ \\
\hline $\begin{array}{l}\text { Querschnittspezifische } \\
\text { Daten (einmalige Eingabe) }\end{array}$ & $\begin{array}{l}\text { - Datum der Erstdiagnose der Querschnittlähmung (QSL) } \\
\text { - Grund der QSL }\end{array}$ \\
\hline $\begin{array}{l}\text { Hospitalisierung (Eingabe } \\
\text { nach jedem Aufenthalt) }\end{array}$ & $\begin{array}{l}\text { - Zentrums-ID (automatisch über angemeldeten Nutzer) } \\
\text { - Aufnahmedatum } \\
\text { - Entlassdatum } \\
\text { - Kostenträger } \\
\text { - Erwerbstätigkeit } \\
\text { - Renten } \\
\text { - Aufnahme von } \\
\text { - Entlassung nach } \\
\text { - Art des Aufenthalts (akut/chronisch (>1 J. nach QSL)) } \\
\text { - Erstaufnahme/Wiederaufnahme } \\
\text { - Komorbiditäten mit negative Einfluss auf das Behandlungsergebnis } \\
\text { - Aufnahmediagnose (wo immer möglich basierend auf ICD-10) } \\
\text { - Hauptdiagnose } \\
\text { - Priorisierte, erste drei Nebendiagnosen }\end{array}$ \\
\hline $\begin{array}{l}\text { Neurologischer Status } \\
\text { (Eingabe nach jedem } \\
\text { Aufenthalt) }\end{array}$ & $\begin{array}{l}\text { - Ausgewählte Parameter der International Standards for Neurological Classification of Spinal Cord Injury (ISNCSCI) [2] } \\
\text { bei Aufnahme und Entlassung } \\
\text { - Segmentale Motorscores } \\
\text { - Sensibles, motorisches und neurologisches Niveau } \\
\text { - ASIA Impairment Scale (AIS) } \\
\text { - Zonen der partiellen Erhaltung (ZPPs) }\end{array}$ \\
\hline $\begin{array}{l}\text { Funktioneller Status } \\
\text { (Eingabe nach jedem } \\
\text { Aufenthalt) }\end{array}$ & $\begin{array}{l}\text { - Alle Items des Spinal Cord Independence Measure (SCIM) III [3] bzw. bei chronischen Patienten des SCIM III } \\
\text { Self-Report [4] bei Aufnahme und Entlassung } \\
\text { - Selbstversorgung } \\
\text { - Atmung und Sphinkter-Management } \\
\text { - Mobilität (zu Hause und Toilette) } \\
\text { - Mobilität (innerhäuslich und außerhäuslich) }\end{array}$ \\
\hline $\begin{array}{l}\text { Lebensqualität (Eingabe } \\
\text { nach jedem Aufenthalt) }\end{array}$ & $\begin{array}{l}\text { - World Health Organization Quality of Life Group - Abbreviated Version (WHOQOL-BREF) [6] bei Aufnahme und } \\
\text { Entlassung }\end{array}$ \\
\hline
\end{tabular}

DMGP nominiert, die sich mit allen Belangen der Registerkonzeptionierung, -implementierung und des Betriebs, aber auch der Nutzung und des Zugriffs auf ParaReg-Daten befasst ( $>$ Abb. 2).

Die Rechte und Pflichten der ParaReg-Exekutivgruppe, welche am Universitätsklinikums Heidelberg für die Implementierung und den Betrieb von ParaReg verantwortlich ist, werden in einem Kooperationsvertrag zwischen der Universität Heidelberg und der DMGP geregelt. Eine zusätzliche Datennutzungsvereinbarung regelt die Prozesse Berichtswesen und Datenexport. Die ParaReg-Exekutivgruppe ist für die Beauftragung des ParaReg-Hosting und einer Treuhandstelle für die Verarbeitung der personenidentifizierenden Daten zuständig. Mit diesen wurden Auftragsdatenverarbeitungsverträge und ggfs. Kooperationsverträge geschlossen.

Daten werden von allen in der DMGP organisierten deutschen Querschnittzentren gesammelt. Als vertragliche Grundlage der freiwilligen Zusammenarbeit dient eine Qualitätssicherungsver- einbarung (QSV), die vom DMGP-Vorstand und dem Leiter bzw. gesetzlichen Vertreter des Zentrums unterzeichnet wird. Die QSV stellt keinen offiziellen Vertrag dar, sondern eine Vereinbarung zur gegenseitigen Zusammenarbeit. Die datenerfassenden Zentren haben das Recht auf vollen Zugriff auf ihre eigenen Daten, wobei nähere Details ein gesonderter Datennutzungsvertrag mit der DMGP regelt.

Wissenschaftler (einschließlich der datenerhebenden Zentren und der ParaReg-Exekutivgruppe) können einen Antrag auf Auswertung von Registerdaten stellen. Der Antrag muss an das ParaReg-Leitungskomitee übermittelt werden, welches diesen auf Basis der wissenschaftlichen Stringenz des Exposés, der inhaltlichen Kriterien und der rechtlichen Zulässigkeit prüft. Im Falle der Annahme wird ein Datennutzungsvertrag zwischen Antragsteller und DMGP geschlossen, welcher Details zu den Datennutzungsrechten, zu treffenden Datenschutzmaßnahmen gemäß Datenschutzgrund- 


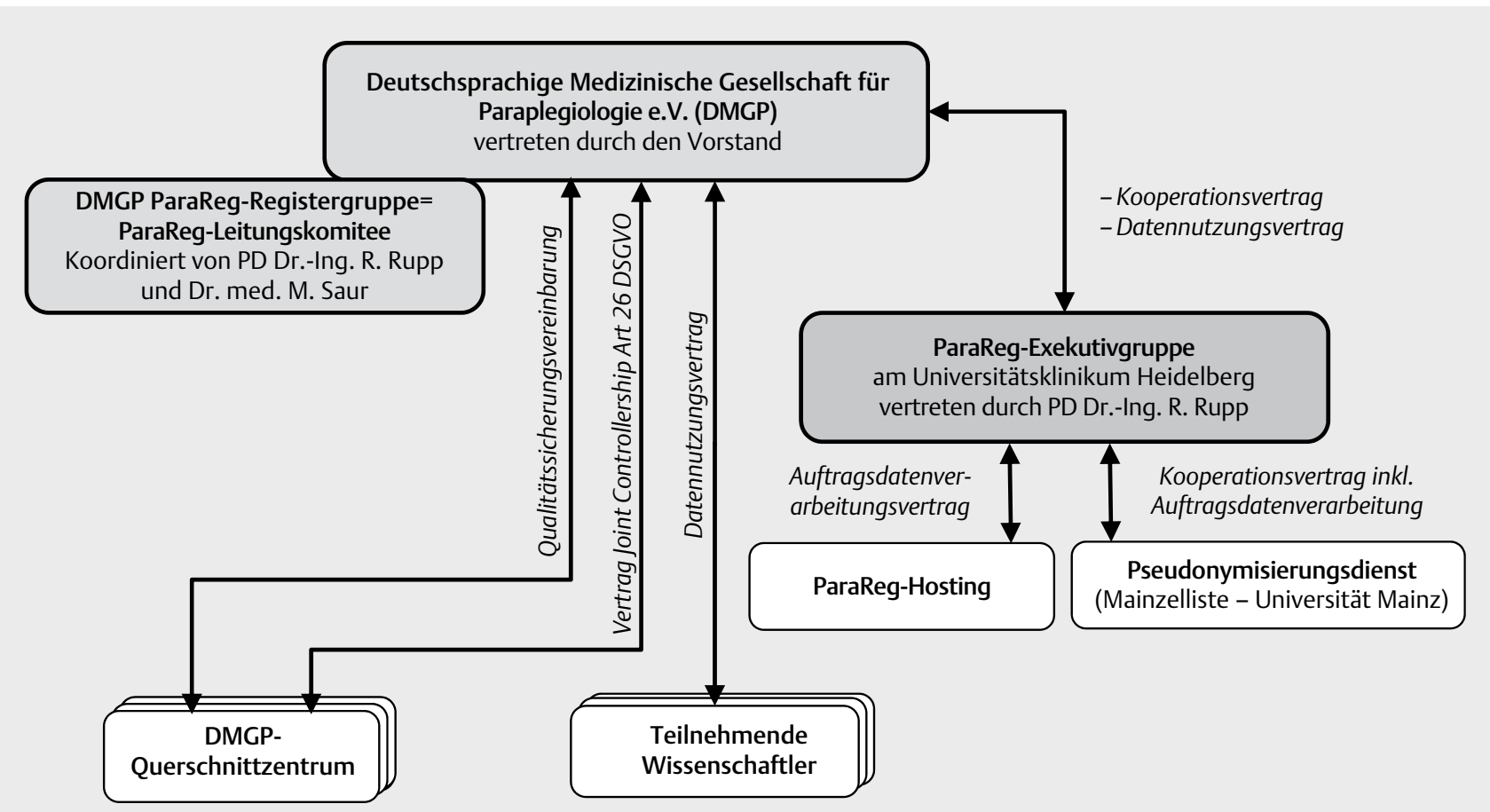

-Abb. 2 Übersicht der Organisationsstuktur des ParaReg-Registers.

verordnung (DSGVO), zu Veröffentlichungsrichtlinien sowie Haftungs- und Gewährleistungsfragen beinhaltet.

\section{Ergebnisse}

Das Pseudonymisierungskonzept von ParaReg basiert auf den Empfehlungen von OSSE, welche sich wiederum an dem klinischen IDManagement-Modul der Technologie- und Methodenplattform für die vernetzte medizinische Forschung e.V. (TMF) orientieren [7]. Dieses klinische Modul besitzt für den Register-Anwender den Vorteil, dass ein Patient nicht über Eingabe einer abstrakten ID, sondern über personenidentifizierende Daten (Vor-/Nachname, Geburtsdatum/-ort, ggfs. aktueller Wohnort) ausgewählt werden kann. Dieses anwenderfreundliche Konzept stellt allerdings spezielle Anforderungen an eine strikte Trennung der Verarbeitung von personenidentifizierenden Daten (IDAT)) und der über die MasterPatienten-ID zugeordneten medizinischen Daten (MDAT), wobei IDAT und MDAT nur im Browser des Anwenders mittels temporärer IDs zusammengefügt werden dürfen. Für die Verwaltung von Master-ID und IDAT wird bei ParaReg eine Instanz der Mainzelliste (http://mainzelliste.de) verwendet [8], die vom Institut für Medizinische Biometrie, Epidemiologie und Informatik (IMBEI) der Universitätsmedizin Mainz gehostet, betrieben und administriert wird.

\section{Datenschutzrechtliche und ethische Voraussetzungen}

Während der Konzeptionierungsphase wurde angelehnt an die OSSE-Empfehlungen ein Datenschutzkonzept und eine Patienteninformationsschrift inklusive -einwilligung erstellt, deren DSGVOKonformität im Januar 2020 durch die AG Datenschutz der TMF, der
Datenschutzbeauftragten des Universitätsklinikums Heidelberg und des Datenschutzkonzernbeauftragten der Berufsgenossenschaften, von denen 8 der 27 deutschen Querschnittzentren betrieben werden, bestätigt wurde. Eine Freigabe hinsichtlich berufsethischer und -rechtlicher Aspekte durch die Ethikkommission der Universität Heidelberg (S-574/2019) erfolge 2019. Zweitvoten liegen mittlerweile von den Landesärztekammern Baden-Württemberg und Hessen und der Universität Bochum vor, weitere sind beantragt.

\section{Datenspeicherung}

Für die Verarbeitung der IDAT wird in ParaReg eine vom IMBEI der Universität Mainz betriebene Mainzelliste-Instanz verwendet. Bei der Auswahl des Hosting-Providers für die MDAT wurden ausschließlich solche mit Firmensitz und Serverstandort in Deutschland zur Wahrung einer maximalen DSGVO-Konformität (Stichwort „Patriot Act“) in Betracht gezogen. Im September 2019 wurde ein ausreichend dimensionierter (Hexa-Core, 64GB Ram, 240 GB SDD, 2x8 TB HDD), dedizierter Server bei der Hetzner Online $\mathrm{GmbH}$ (Gunzenhausen) am Standort Falkenstein angemietet. Weiterhin wurde der gesamte Daten- und Systemspeicher unter Verwendung des Linux Unified Key Setup (LUKS) verschlüsselt, um maximale Datensicherheit bei Entwendung oder unsachgemäßer Entsorgung des physischen Datenträgers zu erreichen.

Zum Schutz auf niedriger Zugriffsebene wurde eine Firewall aufgesetzt, welche lediglich SSH-, HTTP- und HTTPS-Verbindungen zulässt und sämtliche andere Ports blockiert. Als Betriebssystem wird der „stable“-Zweig von Debian (aktuell „Buster“) in der jeweils aktuellsten Version verwendet. Einen Administrationszugang zu diesem System besitzt ausschließlich das ParaReg-Entwick- 
lungsteam, wobei die Zugangsdaten dem Vorstand der DMGP bekannt sind.

\section{Registerimplementierung}

Bei der Auswahl der Softwarepakete zum Betrieb und der Entwicklung des ParaReg-Registers stand die langfristige Verfügbarkeit im Vordergrund, so dass bevorzugt etablierte Open-Source Softwarepakete Anwendung fanden. Auf dem MDAT-Server werden mehrere dedizierte Linux Container (LXC) angelegt, damit Programme installiert und ohne Einfluss auf das restliche System betrieben werden können. Um Änderungen der Datenbankinhalte und dem Export von Daten über die Zeit hinweg zu verfolgen, wurde der MariaDB-Datenbank-Server um ein Audit Plugin ergänzt. Damit können die Vorgaben der DSGVO hinsichtlich Informationspflicht gegenüber den ParaReg-Studienteilnehmern erfüllt werden.

Initial war vorgesehen, das ParaReg-Register unter Verwendung und Erweiterung des OSSE-Frameworks zu implementieren. Allerdings wurden während der Sondierungsphase konzeptionelle Schwierigkeiten z. B. bei der Definition einer zentrumsübergreifenden Master-ID festgestellt, so dass für ParaReg ein speziell an die Bedürfnisse angepasstes System unter dem Python-basierten „Flask“-Framework (v.a. SQLAlchemy zur objektrelationale Bindung von Python-Objekten zur Datenbank) aufgebaut wurde [9].

Zur Kommunikation mit der die IDAT verwaltenden Mainzellisten-Instanz wurde die Middleware-Applikation „MzIClient“ entworfen. MzIClient erlaubt die serverseitige Kommunikation der ParaReg-Applikation mit der Mainzelliste über ein Python-Interface, ohne sich um die tiefere Protokollebenen kümmern zu müssen. Client-seitig liegen korrespondierende JavaScript-Funktionen vor, um Daten mit der Mainzelliste auszutauschen (• Abb. 3).

\section{Elektronische Case Report Forms (eCRFs)}

Für den Betrieb der auf dem ParaReg-Server gehosteten RegisterHomepage (https://parareg.de) wird der weltweit meist genutzte Open-Source-Webserver Engine-X (NGINX; https://nginx.com) verwendet. Dieser Service verwaltet die Transportverschlüsselung eingehender https-Verbindungen und leitet diese als Reverse Proxy an die ParaReg-Anwendung in einem per Firewall abgeschirmten LXC-Container weiter.

Der Zugang zu den einzelnen Funktionen von ParaReg wird über ein rollenspezifisches Zugriffssystem verwaltet. Auf der ParaRegWebseite selbst wurde neben einer Funktionalität zur Auflistung von Patienten und Aufenthalten auch eCRFs zur Registrierung neuer und Verwaltung bestehender Studienteilnehmer, und zur Eingabe der Registervariablen implementiert.

Die eCRFs wurden mit Fokus auf Nutzerfreundlichkeit (UserCentered Design) entwickelt und in einer Alpha-Testphase unter Berücksichtigung der Rückmeldungen von Nutzern angepasst. Im Rahmen der monozentrischen, am Querschnittzentrum in Heidelberg durchgeführten Alpha-Testphase wurden die Aufenthalte von 40 Patienten dokumentiert. Auf der Basis der Rückmeldungen wurden Verbesserungen an der Ergonomie sowie des schnellen Erkennens von unvollständigen Eingaben vorgenommen. Weiterhin wurden alle Formulare mit einer automatischen Speicherfunktion per asynchroner Datenübertragung (Asynchronous JavaScript and XML (AJAX)) ausgestattet. Ein „responsive Webdesign“ sorgt für die nutzerfreundliche Anwendbarkeit von ParaReg unabhängig vom Ein- gabegerät. Die einzelnen Elemente der Website werden an die Bildschirmgröße des Endgerätes wie Smartphones und Tabletcomputer adaptiert und bei Bedarf automatisch angeordnet ( $\mathbf{A b b} \mathbf{\text { 4}}$ ).

\section{Berichtsfunktionalitäten}

Aggregierte Registerdaten können in Standardberichten in Textund Grafikform aufbereitet werden. Dazu zählen Übersichten zu den Patientencharakteristika (z. B. Anteil Tetra-zu Paraplegie, Anteile der verschiedenen Ätiologien, Alters- und Geschlechtsverteilungen) eines einzelnen Zentrums, aber auch übergeordnete Jahresberichte, die auf der DMGP-Homepage veröffentlich werden. Berichte mit Daten eines einzelnen Patienten des eigenen Zentrums können bei Patientenanfragen weitergegeben werden, aber auch als exportierbare Textblöcke für die Entlassbriefdokumentation im Rahmen des Entlassungsmanagements verwendet werden. Mit dieser im klinischen Alltag nützlichen Funktionalität soll die Compliance der ParaReg-Anwender zum vollständigen Eintrag von Aufenthaltsdaten erhöht werden.

\section{Diskussion}

Bisher gibt es in Deutschland zwei wesentliche Datenerhebungen zu Querschnittlähmung: Die bisher einzige Datensammlung, die grundlegende epidemiologische Aspekte von Querschnittgelähmten in Deutschland erfasst (z. B. Anzahl von Patienten mit Tetra-/ Paraplegie, Ursachen des Querschnittlähmung, Anzahl Erstbehandlungen und Wiederaufnahmen), wird seit 1976 vom Querschnittgelähmten-Zentrum am BG Klinikum Hamburg betrieben [10]. Diese Datenerhebung sammelt allerdings nur Basisdaten auf Zentrumsebene und erlaubt im Gegensatz zu ParaReg keine Aussagen über patientenindividuelle Verläufe.

Die zweite Datensammlung wurde 2001 mit der European Multicenter Study about Spinal Cord Injury (EMSCl; http://emsci.org/) initiiert, bei der an mittlerweile 30 europäischen Querschnittzentren (15 aus Deutschland) neurologische und funktionelle Verlaufsdaten während des ersten Jahres nach traumatischer oder ischämischer Querschnittlähmung zu vordefinierten, auf das Verletzungsdatum bezogenen Zeitfenstern erhoben werden [11]. Im Gegensatz zu ParaReg werden in EMSCl aber keine Daten zu Komorbiditäten, Komplikationen und Langzeitverläufe dokumentiert. Darüber hinaus werden in EMSCI hauptsächlich Patienten mit traumatischer Querschnittlähmung eingeschlossen, obwohl heutzutage die Mehrheit der Querschnittlähmungen eine nicht-traumatische Ursache hat. Strukturierte Daten zu nicht-traumatischen Krankheitsentitäten des Rückenmarks sind zum jetzigen Zeitpunkt weder in Deutschland noch weltweit verfügbar.

Auf internationaler Ebene existieren neben EMSCI drei weitere große Register zur Dokumentation von Querschnittgelähmten: Zum einen die seit 1975 bestehende National Spinal Cord Injury Database (NSCID) in den USA [12], die Datenbank des in 2004 gegründete North American Clinical Trials Network (NACTN) [13] und die seit 2006 aktive kanadische Rick Hansen Spinal Cord Injury Registry (RHSCIR) [14] ( Tab. 2). Während sich die Datenerhebung von NACTN wie EMSCI auf das erste Jahr nach Rückenmarkverletzung beschränkt, werden sowohl in der NSCID als auch dem RHSCIR Langzeitverläufe dokumentiert. Auch wenn in diesen beiden Registern die Follow-up Daten hauptsächlich mittels Interviews er- 


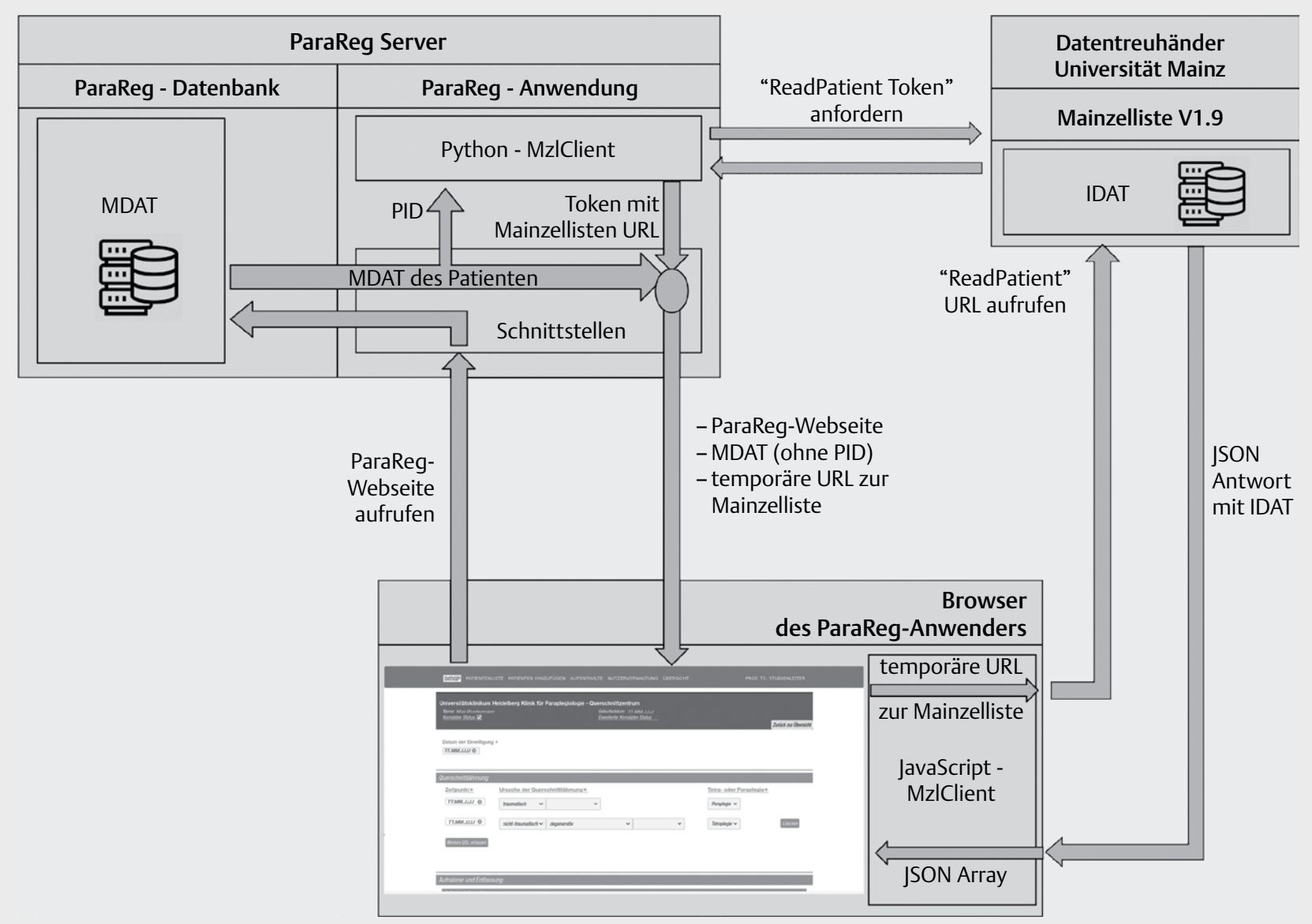

\begin{abstract}
-Abb. 3 Übersicht des Datenflusses zur DSGVO-konformen Zusammenführung von identifizierenden (IDAT) und medizinischen Daten (MDAT) im Browser des ParaReg-Anwenders. MDAT werden direkt aus der Datenbank des ParaReg-Servers ausgelesen. Um die MDAT anhand der vom Anwender im Browser eingegeben IDAT zuzuweisen, wird mittels der zentralen Patienten-ID (PID) des Patienten ein Token von der Mainzelliste durch den Python-MzIClient vom ParaReg-Server angefragt. Dieses Token, eingebettet in die URL der Mainzelliste, wird zusammen mit den MDAT an den Browser übergeben. Über den JavaScript-MzIClient wird die angefertigte URL abgefragt, um die IDAT des Patienten zu erhalten. IDAT sowie MDAT werden anschließend auf der Webseite im Browser des Anwenders dargestellt. JSON: JavaScript Object Notation.
\end{abstract}

hoben werden, ist langfristig ein Datenvergleich mit ParaReg geplant, um Unterschiede in der Effektivität der jeweiligen Gesundheitssysteme identifizieren.

Allen größeren internationalen Registern ist gemeinsam, dass im Wesentlichen nur Menschen mit einer traumatischen Querschnittlähmung eingeschlossen werden. Daher bietet sich mit ParaReg erstmalig die Möglichkeit, zentrumsübergreifend patientenindividuelle Langzeitverlaufsdaten von Querschnittgelähmten nicht nur mit traumatischer, sondern vor allem auch mit nicht-traumatischer Ursache zu sammeln und zu analysieren. Zum gegenwärtigen Stand, hat nach Abschluss der monozentrischen AlphaTestphase die multizentrische Datenerhebung an den 5 Querschnittzentren der ParaReg-Lenkungsausschusses begonnen, die im Laufe der Jahre 2021/22 auf alle 27 DMGP-Querschnittzentren ausgeweitet werden soll. Von technischer Seite wird die Möglichkeit zur Integration von gerätegestützt erhobenen Daten von Smartsensors/-phones wie Aktivitätsdaten oder Patiententagebücher geschaffen. Dies eröffnet die Möglichkeit einer kontinuierlichen, außerklinischen Verlaufsdokumentation und eine spätere
Korrelation mit Komplikationshäufigkeiten. Das ParaReg-Register ist als Langzeitregister für den Betrieb über mehrere Jahrzehnte ausgelegt, dessen Erhalt und Nachhaltigkeit durch die Schirmherrschaft und Führung des Registers durch die DMGP-Fachgesellschaft hinaus gewährleistet ist. Mittelfristig ist sowohl IT-technisch als auch von den ethisch-rechtlichen Rahmenbedingungen eine Integration des EMSCI-Registers in das DSGVO-kompatible ParaRegRegister geplant. In einem ersten Schritt in diese Richtung wurde eine Mehrsprachenunterstützung der ParaReg-Eingabemasken implementiert.

Bei der Implementierung des ParaReg-Register traten bisher einige besondere Herausforderungen auf: Der Aufwand und Zeitbedarf zur Schaffung der rechtlichen Voraussetzungen mit der Erstellung und rechtlichen Prüfung von Kooperations- und Datenverarbeitungsverträgen war wesentlich höher als ursprünglich angenommen. Bei den berufs- und datenschutzrechtlichen Beratungen bereiteten teilweise widersprüchliche Auflagen bzw. Empfehlungen große Probleme. Hier wäre die Schaffung der Möglichkeit zu einer zentralen Begutachtung sehr hilfreich. Bei der techni- 

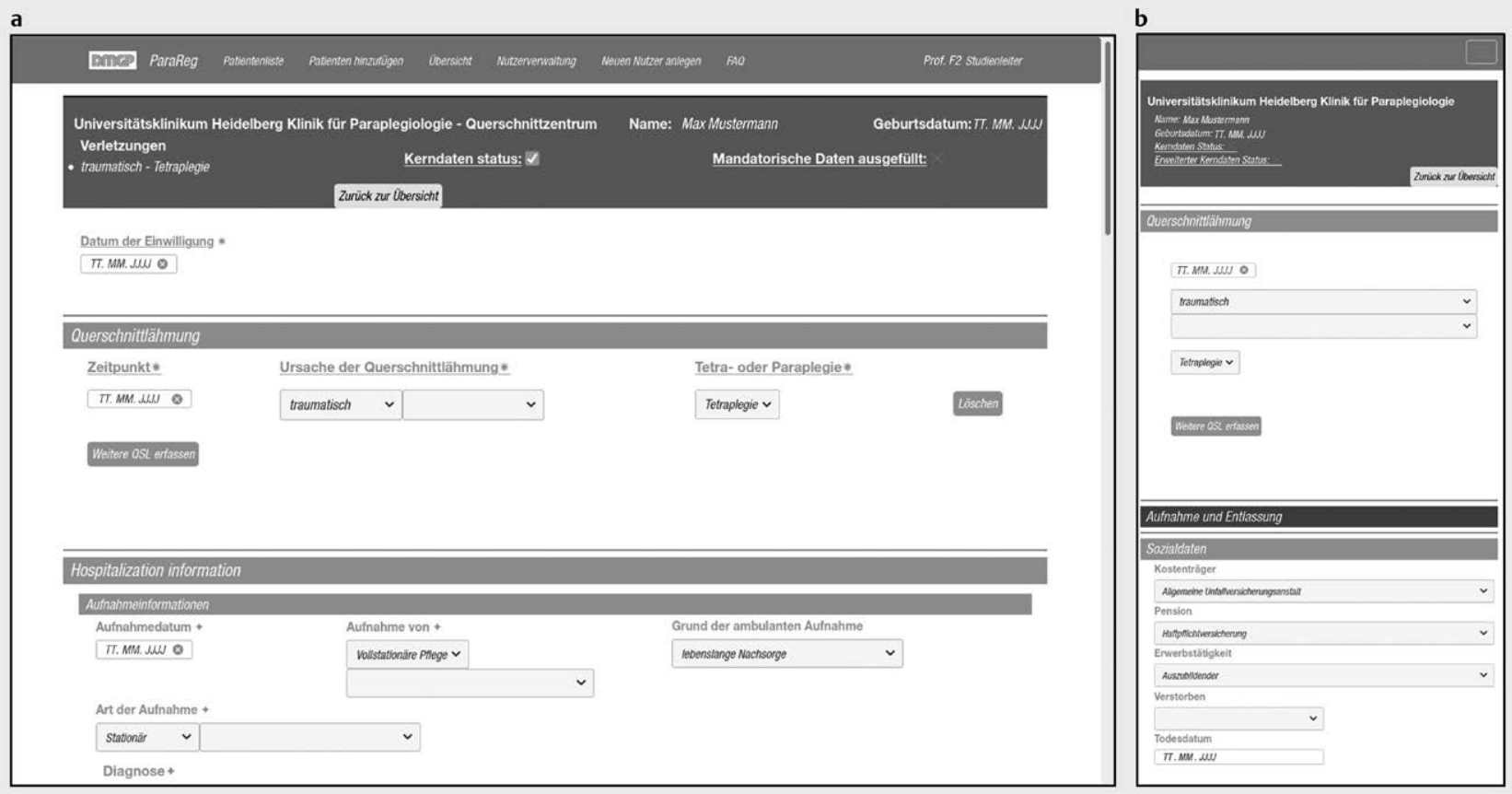

Abb. 4 Elektronische ParaReg Case Report Forms (eCRFs) zur Dokumentation eines Krankenhausaufenthaltes. Linke Teilabbildung a: Ansicht der Haupteingabeseite auf einem Standard-PC. Rechte Teilabbildung b: Ansicht der Haupteingabeseite auf einem Tablet-Computer.

> Tab. 2 Übersicht von internationalen Registern zur Dokumentation von Querschnittgelähmten und deren wesentlichen Kenndaten (Stand 2020).

\begin{tabular}{|c|c|c|c|c|}
\hline & $\begin{array}{l}\text { Model Spinal Cord Injury } \\
\text { System (MSCIS), USA [12] }\end{array}$ & $\begin{array}{l}\text { North American } \\
\text { Clinical Trials } \\
\text { Network (NACTN), } \\
\text { USA [13] }\end{array}$ & $\begin{array}{l}\text { Rick Hansen Spinal Cord } \\
\text { Injury Registry (RHSCIR), } \\
\text { Kanada [14] }\end{array}$ & $\begin{array}{l}\text { European Multicenter- } \\
\text { Study about Spinal Cord } \\
\text { Injury (EMSCI), Europa } \\
\text { [11] }\end{array}$ \\
\hline Gründungsjahr & 1975 & 2006 & 2004 & 2001 \\
\hline Patientenpopulation & $\begin{array}{l}\text { Akute traumatische } \\
\text { Rückenmarkverletzungen }\end{array}$ & $\begin{array}{l}\text { Akute traumatische } \\
\text { Rückenmarkverletzun- } \\
\text { gen }\end{array}$ & $\begin{array}{l}\text { Akute traumatische } \\
\text { Rückenmarkverletzungen }\end{array}$ & $\begin{array}{l}\text { Akute traumatische und } \\
\text { ischämische Querschnitt- } \\
\text { lähmungen }\end{array}$ \\
\hline $\begin{array}{l}\text { Zeitpunkte Assessments } \\
\text { Erstbehandlung }\end{array}$ & Aufnahme und Entlassung & $\begin{array}{l}\text { Aufnahme und } \\
\text { Entlassung }\end{array}$ & $\begin{array}{l}\text { Aufnahme und Entlassung } \\
\text { Primärzentrum und } \\
\text { zusätzlich Erstreha }\end{array}$ & $\begin{array}{l}<4 \text { Wo., 4, 12, 24, } 48 \text { Wo. } \\
\text { nach Eintritt der } \\
\text { Querschnittlähmung }\end{array}$ \\
\hline $\begin{array}{l}\text { Zeitpunkte Assessments } \\
\text { Follow-up }\end{array}$ & $\begin{array}{l}1 \text { und } 5 \text { Jahre, danach } \\
\text { lebenslang alle } 5 \text { Jahre }\end{array}$ & $\begin{array}{l}6 \text { und } 12 \text { Mon. nach } \\
\text { Eintritt der Quer- } \\
\text { schnittlähmung }\end{array}$ & $\begin{array}{l}\text { 1, } 2 \text { und } 5 \text { Jahre, danach } \\
\text { lebenslang alle } 5 \text { Jahre }\end{array}$ & Kein Follow-up \\
\hline Anzahl Variablen & $\begin{array}{l}\text { Erstbehandlung: } 682 \\
\text { Interview-basierter } \\
\text { Follow-up: } 339\end{array}$ & $\begin{array}{l}\text { Erstbehandlung: nicht } \\
\text { bekannt } \\
\text { Follow-up: } 39\end{array}$ & $\begin{array}{l}\text { Erstbehandlung und -reha: } \\
256 \\
\text { Interview-basierter } \\
\text { Follow-up: } 102\end{array}$ & Bis zu 1020 \\
\hline $\begin{array}{l}\text { Anzahl aktiv } \\
\text { datenerhebende Zentren }\end{array}$ & 14 (+ 5 nur follow-up) & 8 & 15 & 23 \\
\hline $\begin{array}{l}\text { Anzahl dokumentierte } \\
\text { Patienten }\end{array}$ & 34734 (Sept. 2020) & $>1200$ (Ende 2017) & 7800 (Ende 2019) & 5006 (Ende 2019) \\
\hline $\begin{array}{l}\text { Finanzielle } \\
\text { Unterstützung }\end{array}$ & $\begin{array}{l}\text { National Institute on } \\
\text { Disability, Independent } \\
\text { Living, and Rehabilitation } \\
\text { Research (NIDILRR), } \\
\text { Washington DC, USA }\end{array}$ & $\begin{array}{l}\text { Christopher and Dana } \\
\text { Reeve Foundation, } \\
\text { Short Hills, NJ, USA }\end{array}$ & $\begin{array}{l}\text { Praxis Institute, Vancouver, } \\
\text { Kanada }\end{array}$ & Kein externes Funding \\
\hline
\end{tabular}


schen Implementierung hat die konsequente Umsetzung der Patientenrechte nach DSGVO hinsichtlich Dateneinsicht, Datenlöschung und ggfs. Datenzerstörung auch aus Backups ähnlich viele Ressourcen beansprucht wie die Programmierung der gesamten elektronischen Dateneingabeformulare.

\section{Danksagung}

Das vorgestellte Projekt wird mit Mitteln des Bundesministeriums für Bildung und Forschung (BMBF) unter dem Förderkennzeichen 01GY1904 und der Deutschsprachigen Medizinischen Gesellschaft für Paraplegiologie e.V. (DMGP) gefördert.

\section{Interessenkonflikt}

Die Autorinnen/Autoren geben an, dass kein Interessenkonflikt besteht. Die Autorinnen/Autoren erhalten eine finanzielle Förderung vom BMBF, der DMGP und des Universitätsklinikums Heidelberg.

\section{Literaturverzeichnis}

[1] Deutschsprachige Gesellschaft für Paraplegiologie e.V., Deutsche Stiftung Querschnittlähmung, Deutscher Rollstuhl-Sportverband et al. Pressemappe Querschnittlähmung. Im Internet: https://dmgp.de/ images/presse/DMGP-DRS-DSQ-FGQ-Pressemappe_online.pdf

[2] Rupp R, Biering-Sørensen F, Burns SP et al. International Standards for Neurological Classification of Spinal Cord Injury: Revised 2019. Top Spinal Cord Inj Rehabil 2021; 27: 1-22

[3] Itzkovich M, Gelernter I, Biering-Sorensen F et al. The Spinal Cord Independence Measure (SCIM) version III: reliability and validity in a multi-center international study. Disabil Rehabil 2007; 29: 1926-1933
[4] Fekete C, Eriks-Hoogland I, Baumberger M et al. Development and validation of a self-report version of the Spinal Cord Independence Measure (SCIM III). Spinal Cord 2013; 51: 40-47

[5] Biering-Sorensen F, Charlifue S, DeVivo M et al. International Spinal Cord Injury Data Sets. Spinal Cord 2006; 44: 530-534

[6] Skevington SM, Lotfy M, O'Connell KA et al. The World Health Organization's WHOQOL-BREF quality of life assessment: psychometric properties and results of the international field trial. A report from the WHOQOL group. Qual Life Res 2004; 13: 299-310

[7] Pommerening K, Drepper J, Helbing K et al. Leitfaden zum Datenschutz in medizinischen Forschungsprojekten. Generische Lösungen der TMF 2.0. Berlin: Medizinisch Wissenschaftliche Verlagsgesellschaft; 2014

[8] Lablans M, Borg A, Uckert F. A RESTful interface to pseudonymization services in modern web applications. BMC Med Inform Decis Mak 2015; 15 : 2

[9] Grinberg M. Flask Web Development. 2. Aufl. O'Reilly Media Inc; 2018

[10] Exner G. Der Arbeitskreis „Querschnittlähmungen“ des Hauptverbandes der gewerblichen Berufsgenossenschaften in Deutschland. Fakten-Zahlen-Prognosen. Trauma und Berufskrankheit 2004; 6: 147-150

[11] Curt A, Schwab ME, Dietz V. Providing the clinical basis for new interventional therapies: refined diagnosis and assessment of recovery after spinal cord injury. Spinal Cord 2004; 42: 1-6

[12] Chen Y, DeVivo MJ, Richards JS et al. Spinal Cord Injury Model Systems: Review of Program and National Database From 1970 to 2015. Arch Phys Med Rehabil 2016; 97: 1797-1804

[13] Grossman RG, Toups EG, Frankowski RF et al. North American Clinical Trials Network for the Treatment of Spinal Cord Injury: goals and progress. J Neurosurg Spine 2012; 17: 6-10

[14] Noonan VK, Kwon BK, Soril L et al. The Rick Hansen Spinal Cord Injury Registry (RHSCIR): a national patient-registry. Spinal Cord 2012; 50: 22-27 